EDUCAÇÃO

V.9 • N.2 • 2020 - Número Temático

ISSN Digital: 2316-3828

ISSN Impresso: 2316-333X

DOI: 10.17564/2316-3828.2020v9n2p51-65
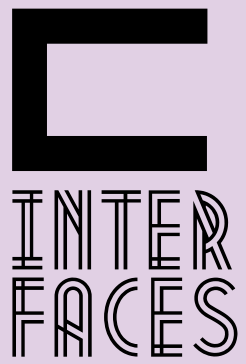

CIENTIIFICAS

\section{O PENSAMENTO ARITMÉTICO, SUAS RELAÇ̃̃ES COM AS FUNÇÕES EXECUTIVAS E AS CONTRIBUIÇÕES DOS JOGOS DIGITAIS: UM ESTUDO COM ALUNOS UNIVERSITÁRIOS}

THE ARITHMETIC THOUGHT, ITS RELATIONS WITH THE EXECUTIVE FUNCTIONS AND THE CONTRIBUTIONS OF DIGITAL GAMES: A STUDY WITH UNIVERSITY STUDENTS

\section{EL PENSAMIENTO ARITMÉTICO, SUS RELACIONES CON LAS FUNCIONES EJECUTIVAS Y LAS CONTRIBUCIONES DE LOS JUEGOS DIGITALES: UN ESTUDIO CONESTUDIANTES UNIVERSITARIOS}

William de Souza Santos ${ }^{1}$ Lynn Rosalina Gama Alves ${ }^{2}$

\section{RESUMO}

As funções executivas têm papel fundamental no processo de ensino aprendizagem pelo fato de estarem associadas ao desenvolvimento cognitivo, emocional, comportamental e social. Diante dos grandes déficits que as avaliações nacionais e internacionais vêm apontando sobre o conhecimento matemático dos estudantes brasileiros, uma possível intervenção é a utilização de digital brain games para estimular as funções executivas. Neste intuito, este artigo tem o objetivo de investigar como os $d i$ gital brain games podem contribuir para o aprendizado da matemática e para cumprir este objetivo. Esta pesquisa apresenta um cunho qualitativo de caráter exploratório e contou com um estudo de caso com a participação de 16 alunos universitários. Os resultados demonstram que os digital brain games permitem com que os jogadores reforcem e aprimorem seus conhecimentos em um ambiente lúdico, motivador e desafiador, algo que pode contribuir para a aprendizagem matemática.

\section{PALAVRAS-CHAVE}

Funções Executivas. Jogos Digitais. Matemática. 


\section{ABSTRACT}

Executive functions play a fundamental role in the teaching - learning process because they are associated with cognitive, emotional, behavioral and social development. Given the large deficits that national and international evaluations have been pointing to the mathematical knowledge of Brazilian students, a possible intervention is the use of digital brain games to stimulate executive functions. The purpose of this article is to investigate how digital brain games can contribute to the learning of mathematics and to fulfill this objective, this research presents a qualitative exploratory character and had a case study with the participation of 16 students College students. The results demonstrate that digital brain games allow players to reinforce and enhance their knowledge in a playful, motivating and challenging environment, which can contribute to mathematical learning.

\section{KEYWORDS}

Executive Functions. Digital games. Mathematics.

\section{RESUMEN}

Las funciones ejecutivas tienen un papel fundamental en el proceso de enseñanza aprendizaje por el hecho de estar asociadas al desarrollo cognitivo, emocional, comportamental y social. Ante los grandes déficits que las evaluaciones nacionales e internacionales vienen apuntando sobre el conocimiento matemático de los estudiantes brasileños, una posible intervención es la utilización de digital brain games para estimular las funciones ejecutivas. En este sentido, este artículo tiene el objetivo de investigar cómo los digital brain games pueden contribuir al aprendizaje de las matemáticas y para cumplir este objetivo, esta investigación presenta un cuño cualitativo de carácter exploratorio y contó con un estudio de caso con la participación de 16 alumnos de universidad. Los resultados demuestran que los juegos mentales permiten que los jugadores refuercen y perfeccionen sus conocimientos en un ambiente lúdico, motivador y desafiante, algo que puede contribuir al aprendizaje matemático.

\section{PALABRAS CLAVE}

Funciones Ejecutivas. Juegos Digitales. Matemáticas. 


\section{INTRODUÇ̧̃̃O}

Nas últimas duas décadas, as avaliações nacionais e internacionais vêm apontando o déficit do conhecimento matemático dos estudantes brasileiros. Segundo o relatório do Programa de Avaliação Internacional de Estudantes (PISA) (OCDE, 2016), no ano de 2015, os estudantes brasileiros obtiveram 377 pontos, fazendo com que o Brasil ocupe $65^{a}$ posição no ranking desta avaliação. Este mesmo relatório sinaliza que no Brasil, $70,3 \%$ dos estudantes estão abaixo do nível 2 em matemática, patamar que a OCDE estabelece como necessário para que os jovens possam exercer plenamente sua cidadania.

Tal situação também tem sido evidenciada no Índice de Desenvolvimento da Educação Básica (IDEB) de 2015, onde é apontado que somente $16 \%$ dos alunos que saem do $9^{\circ}$ ano têm o nível de proficiência adequado na área de matemática, enquanto apenas $9 \%$ dos jovens que terminam o $3^{\circ}$ ano dominam os conhecimentos básicos sobre a matemática.

Diante destes dados, pode-se constatar que grande parte dos estudantes que ingressarão no ensino superior apresentam grande dificuldade em matemática e isso de certa forma pode impactar na sua formação inicial. Por este motivo, se faz necessário buscar intervenções que possam auxiliar estes estudantes na melhoria das habilidades e conhecimentos matemáticos.

Segundo Gonçalves (2017), as funções executivas são consideradas como as principais preditoras do desempenho escolar e influenciam diretamente a escrita, leitura e aritmética. Por este motivo, é importante compreender o que são as funções executivas, como elas podem influenciar o conhecimento matemático e como as intervenções direcionadas nessas funções podem contribuir na melhoria das habilidades matemáticas.

Autores como Rivero, Quirino e Starling-Alves (2012), Rodrigues, Rivero e Bertalia (2013), Ramos (2013), Alves (2016), Tourinho, Bonfim, Alves (2016), Guimarães, Tourinho, Vieira, Alves (2016), Vieira, Cerqueira e Dorea (2016), Rocha, Nery e Alves (2014), tem pesquisado como a utilização de jogos digitais podem estimular o desenvolvimento cognitivo e as funções executivas de crianças e adolescentes, mas até o momento não se encontram muitos estudos com alunos universitários e nem de como a estimulação das funções executivas pode contribuir de forma concreta para a aprendizagem matemática.

Neste intuito, este artigo tem por objetivo investigar como a estimulação das funções executivas por meio das interações com jogos digitais pode contribuir para o aprendizado matemático dos alunos universitários.

\section{FUNDAMENTAÇÃO TEÓRICA}

As funções executivas são as habilidades cognitivas necessárias para controlar nossos pensamentos, nossas emoções e nossas ações. Segundo Diamond (2013), as funções executivas (FE) têm grande influência sobre o desempenho acadêmico, pelo fato de estarem associadas ao ajustamento e ao desenvolvimento cognitivo, emocional, comportamental e social.

As funções executivas podem ser divididas em: memória de trabalho (responsável pelo armazenamento e atualização das informações durante uma atividade), controle inibitório (responsável pela 
inibição da resposta prepotente em uma tarefa) e flexibilidade mental (capacidade de mudar a postura de atenção e cognição).

Estudos como os de Cragg \& Gilmore (2014), St. Clair-Thompson \& Gathercole (2009), Altemeier, Abbott, \& Berninger (2008), têm mostrado a influência da memória de trabalho, da flexibilidade cognitiva e do controle inibitório no desempenho da leitura, da produção escrita e das habilidades matemáticas.

Nos resultados da pesquisa desenvolvida por Gonçalves (2017), foi identificado que o controle inibitório prediz o desempenho em aritmética de crianças de $1^{\circ}$ a $5^{\circ}$ ano do Ensino Fundamental, enquanto a flexibilidade cognitiva prediz o desempenho de estudantes mais velhos, de $6^{\circ}$ a $9^{\circ}$ ano do Ensino Fundamental. Tal fato já tinha sido sinalizado por Megías \& Macizo (2016) que constataram que o papel do controle inibitório no início do processo de aprendizagem parecia estar relacionado à escolha do fato aritmético a ser utilizado nos diferentes cálculos matemáticos.

De forma similar, Bull \& Lee (2014), identificaram que a flexibilidade cognitiva pôde explicar o desempenho de estudantes mais velhos em cálculos mais complexos, que exigem alternância de procedimentos e regras matemáticas ou, ainda, de estratégias de solução dos desafios matemáticos.

Diante dos resultados apresentados por estes autores, vê-se que a estimulação das funções executivas pode contribuir para melhoria do desempenho da leitura, da produção escrita e das habilidades matemáticas. Entretanto, todos esses estudos apresentados tiveram como público-alvo, alunos do ensino fundamental e não são encontradas muitas pesquisas que envolvam alunos universitários, público este que tem apresentado grandes dificuldades quanto ao conhecimento matemático, que vem impactando na sua aprendizagem e consequentemente na sua formação profissional.

No cenário nacional e internacional, uma das estratégias que vem sendo utilizada para estimular as funções executivas de crianças e adolescentes é a interação com os jogos digitais, já que a presença destes artefatos culturais no cotidiano desta geração tem sido grandiosa.

No Brasil por exemplo, encontramos os trabalhos de Rivero, Quirino e Starling-Alves (2012), Rodrigues, Rivero e Bertalia (2013), que discutem o impacto do videogame no desenvolvimento cognitivo dos adolescentes. Já Oliveira, Ishitani e Cardoso (2013) trazem uma revisão sistemática sobre jogos de computador e Transtorno de Déficit de Atenção e Hiperatividade, que apresenta comprometimento das funções executivas.

Outro trabalho de Rivero (2016), apresentou o desenvolvimento do jogo BIGAME e a investigação do seu potencial para estimular o controle inibitório de adolescentes. De forma similar, integrantes do Centro de Pesquisa Comunidades Virtuais, como por exemplo Alves (2016), Tourinho, Bonfim e Alves (2016), Guimarães, Tourinho, Vieira e Alves (2016), Vieira, Cerqueira e Dorea (2016), Rocha, Nery e Alves (2014), vêm apontando nesses estudos os resultados das interações de alunos do ensino fundamental com o Gamebook Guardiões da Floresta, uma mídia híbrida para estimular as funções executivas de crianças na faixa etária de 8 a 12 anos.

Esta mesma estratégia pode ser adotada com os alunos do ensino superior, na tentativa de minimizar as dificuldades que eles têm apresentado no que se refere a matemática básica, principalmente a base aritmética que tem fundamental importância para o conhecimento matemático. Para tanto se faz necessário identificar como esta intervenção por meio dos jogos digitais pode contribuir na 
melhoria das funções executivas e assim impactar no progresso das habilidades matemáticas dos estudantes universitários.

\section{MATERIAIS E MÉTODOS}

Esta investigação tem um cunho qualitativo, do tipo estudo de caso, realizada com 16 alunos universitários divididos em Grupo A (GA), formado por 9 estudantes do Bacharelado Interdisciplinar em Ciência e Tecnologia (BI-CET) e BI em Humanidades ( $\mathrm{BI}-\mathrm{H}$ ) ingressantes no curso, com idades que variavam de 17 a 24 anos e Grupo B (GB), formado por 7 alunos dos Bls em CeT, H, Artes e Saúde, com variação de idade de 19 a 51 anos.

Os grupos foram formados a partir de duas disciplinas diferentes ministradas pela investigadora e, portanto, a frequência da mediação em sala de aula foi diretamente proporcional aos encontros semanais com os alunos: a disciplina do GA possuía 2 encontros semanais durante a tarde, enquanto o GB apenas 1 encontro a noite. Ao final, as mediações supervisionadas somaram o total de 5 mediações para o GA e 2 mediações para o GB.

A partir de 1 de maio de 2018, iniciou-se a segunda parte onde os alunos de ambos os grupos passaram a realizar as mediações em casa, com o auxílio de um roteiro on-line, onde eles preenchiam os resultados adquiridos nos treinamentos diários até 31/07/2018. A Tabela 1, traz um perfil mais detalhado de cada aluno.

Tabela 1 - Caracterização dos Alunos

\begin{tabular}{|c|c|c|c|}
\hline Identificador & Idade & Gênero & Curso \\
\hline P01 & 18 & Fem & $\mathrm{BI}-\mathrm{CeT}$ \\
\hline P02 & 20 & Fem & $\mathrm{BI}$ - Human. \\
\hline P03 & 17 & Fem & $\mathrm{BI}-\mathrm{CeT}$ \\
\hline P04 & 19 & Fem & $\mathrm{BI}-\mathrm{CeT}$ \\
\hline P05 & 19 & Fem & $\mathrm{BI}-\mathrm{CeT}$ \\
\hline P06 & 21 & Fem & $\mathrm{BI}-\mathrm{CeT}$ \\
\hline $\mathrm{P} 07$ & 18 & Masc & $\mathrm{BI}-\mathrm{CeT}$ \\
\hline P08 & 19 & Fem & $\mathrm{BI}-\mathrm{CeT}$ \\
\hline P09 & 22 & Fem & $\mathrm{BI}-\mathrm{CeT}$ \\
\hline P10 & 51 & Masc & $\mathrm{BI}$ - Human. \\
\hline $\mathrm{P} 11$ & 21 & Masc & $\mathrm{BI}-\mathrm{CeT}$ \\
\hline P12 & 43 & Fem & $\mathrm{BI}$ - Human. \\
\hline $\mathrm{P} 13$ & 37 & Masc & $\mathrm{BI}$ - Artes \\
\hline $\mathrm{P} 14$ & 43 & Masc & BI - Saúde \\
\hline P15 & 23 & Masc & $\mathrm{BI}-\mathrm{CeT}$ \\
\hline $\mathrm{P} 16$ & 21 & Masc & $\mathrm{BI}$ - Artes \\
\hline
\end{tabular}

Fonte: Dados da pesquisa. 
Durante esse período da interação com os jogos do aplicativo Lumosity é importante salientar que os jogos que compõem o treinamento diário são oferecidos aleatoriamente aos jogadores. Por este motivo, a frequência dos jogos nos relatórios diferiu de aluno para aluno, impedindo uma análise comparativa mais aprofundada, por serem muitas vezes aparições pontuais. Foi definido que cada aluno deveria realizar a mediação com o Lumosity no mínimo 3 vezes durante a semana, não importando como este distribuiria as mediações.

\subsection{CARACTERIZAÇÃO DO APP LUMOSITY E DOS JOGOS AVALIADOS}

O App Lumosity é caracterizado por seus desenvolvedores como um programa de treinamento cerebral que consiste em jogos com desafios divertidos e interativos para ajudar a manter sua mente ativa. Ele possui versões para Android e IOS e tem sido usado por mais de 90 milhões de pessoas em todo o mundo, que interagem com jogos educativos de treinamento do cérebro, projetado para testar raciocínio crítico, memória e habilidades de solução de problemas.

São disponibilizados no app mais de 25 jogos mentais que desafiam habilidades cognitivas e acadêmicas, incluindo: jogos de desafio, jogos de memória, jogos de solução de problemas, jogos de lógica, jogos de raciocínio crítico, jogos de matemática etc.

Os jogos avaliados neste artigo são indicados pelo Lumosity como aqueles que estimulam além das funções executivas, conhecimentos aritméticos. 0 primeiro jogo avaliado é denominado de Conta-gotas. Nele, o usuário precisa digitar o resultado das operações fundamentais (adição, subtração, multiplicação e divisão) antes das gotas tocarem o chão, como pode ser visto na Figura 1.

Ao longo do tempo, as operações se misturam entre fáceis e difíceis (com 1 ou 2 algarismos) e a velocidade das gotas que caem aumenta. Neste jogo são necessários conhecimentos sobre as operações matemáticas, velocidade (agilidade) nos cálculos (apesar de não ser apresentado um cronômetro regressivo) e foco.

Figura 1 - Jogo Conta-gotas

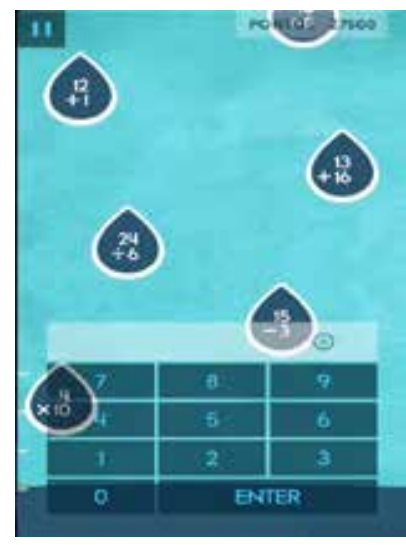


O segundo jogo analisado tem o nome de Desafio do Quadro Negro. Neste jogo o usuário precisa comparar duas expressões numéricas, estabelecendo se elas são iguais ou qual delas é a maior, como pode ser visto na Figura 2. Além dos conhecimentos em operações matemáticas, expressões numéricas é necessário que o jogador tenha velocidade nas respostas, pois o jogo apresenta um cronômetro regressivo), além de indicar o sentido correto (direito ou esquerdo) na tela do seu dispositivo.

Figura 2 - Jogo Desafio do Quadro negro

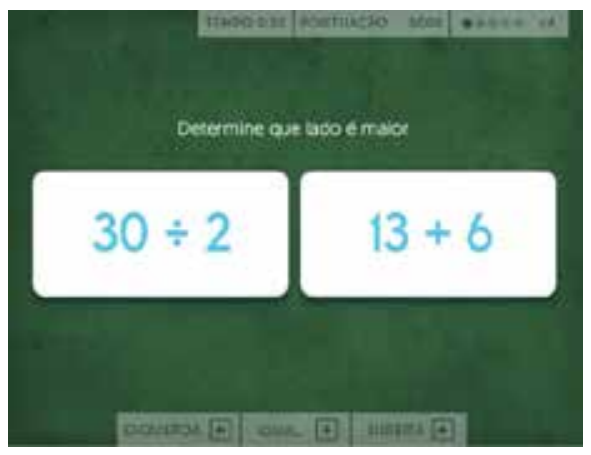

Em suma, estes dois jogos tem como fundamento estimular os conhecimentos aritméticos, o primeiro em um nível menor, evidenciando operações simples e o segundo, expressões que podem envolver parênteses, ambos necessitando de cálculos mentais.

\section{RESULTADOS E DISCUSSÃO}

Ao analisar as interações de 5 indivíduos do Grupo A com o jogo Conta-gotas, 3 deles mostraram evolução na pontuação, o que indica uma maior quantidade de acertos e de tempo de interação. Deles, o indivíduo P04 apresenta um maior domínio nas operações, já que sua pontuação se comparada aos demais, apresenta escores muito mais elevados, como pode ser visto na Figura 3.

Este aumento nas pontuações demonstra que os jogadores apresentaram um maior repertório de conhecimentos aritméticos. Observando os jogadores P01 e P06, vê-se que eles apresentaram maiores dificuldades na segunda interação, demonstrando que eles tiveram dificuldades quanto às operações sugeridas pelo jogo, já que os mesmos obtiveram pontuações menores nesta interação. 
Figura 3

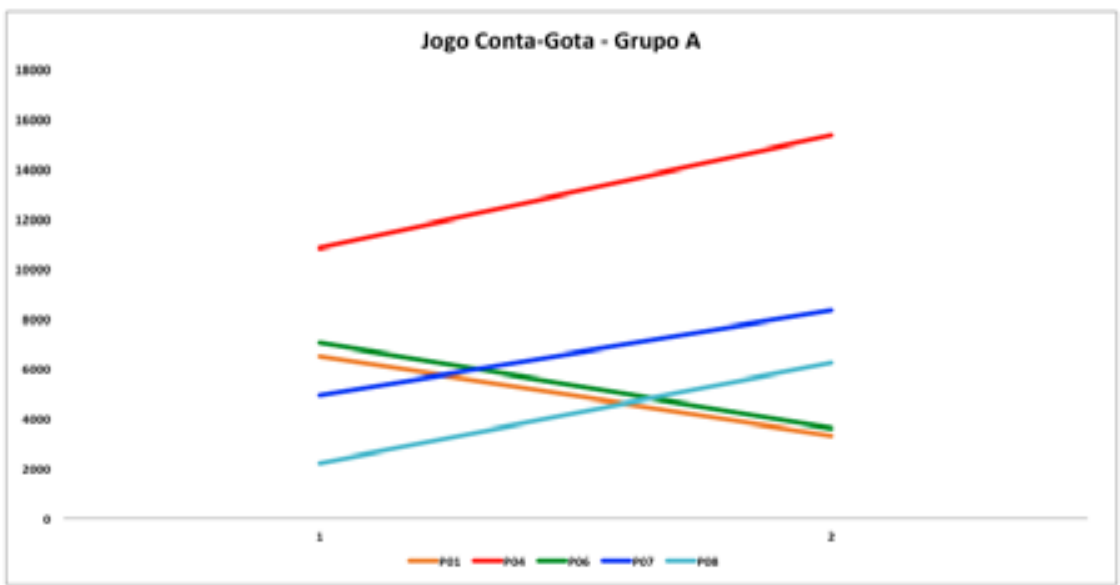

Ao analisar os dados das interações dos indivíduos do Grupo B com o mesmo jogo (FIGURA 4), observa-se que o gráfico apresenta maior instabilidade nas pontuações, onde os jogadores demonstram altos e baixos, com exceção do indivíduo P13, que em suas interações demonstrou apenas avanços na pontuação. A maior inconstância nas interações é registrada pelo indivíduo P16 que nas interações 4 e 5 apresentou escores muito baixos, o que pode ser reflexo de dificuldades com alguma operação em específico.

\section{Figura 4}

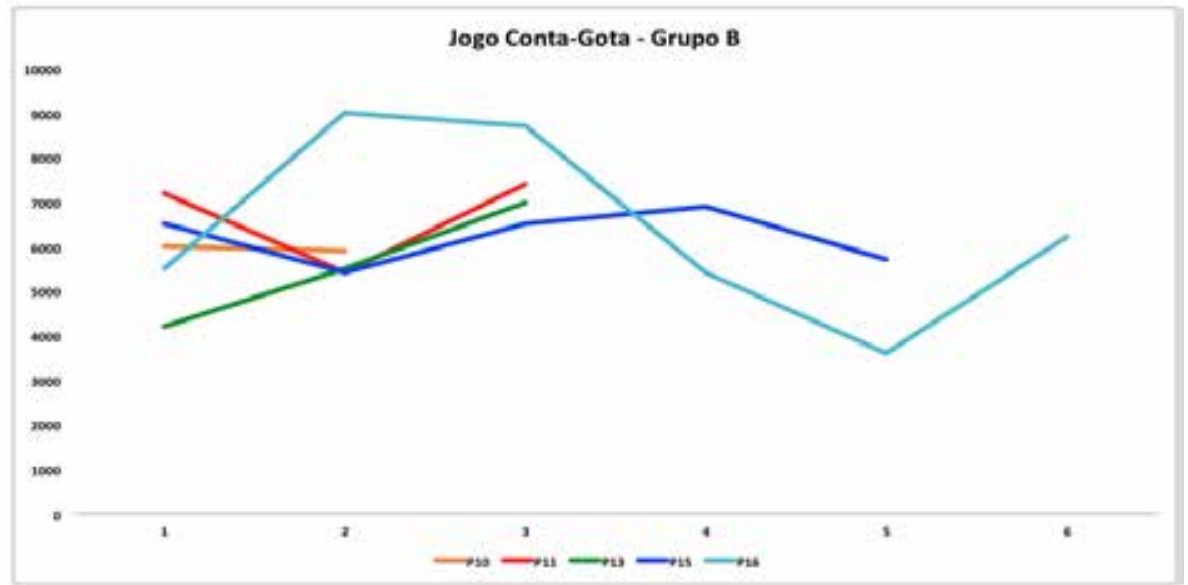


Podemos perceber que, comparando os dois grupos (FIGURA 5), o indivíduo P04 apresenta os melhores escores entre os alunos, apontando que este possui um maior conhecimento nas operações fundamentais. Os demais jogadores apresentam perfis similares, demonstrando uma regularidade no conhecimento das operações fundamentais, com exceção dos jogadores P01 e P06 que diminuíram, em muito, suas pontuações, refletindo que eles possuem certa dificuldade no cálculo das operações ou na velocidade de realizarem o cálculo mental.

\section{Figura 5}

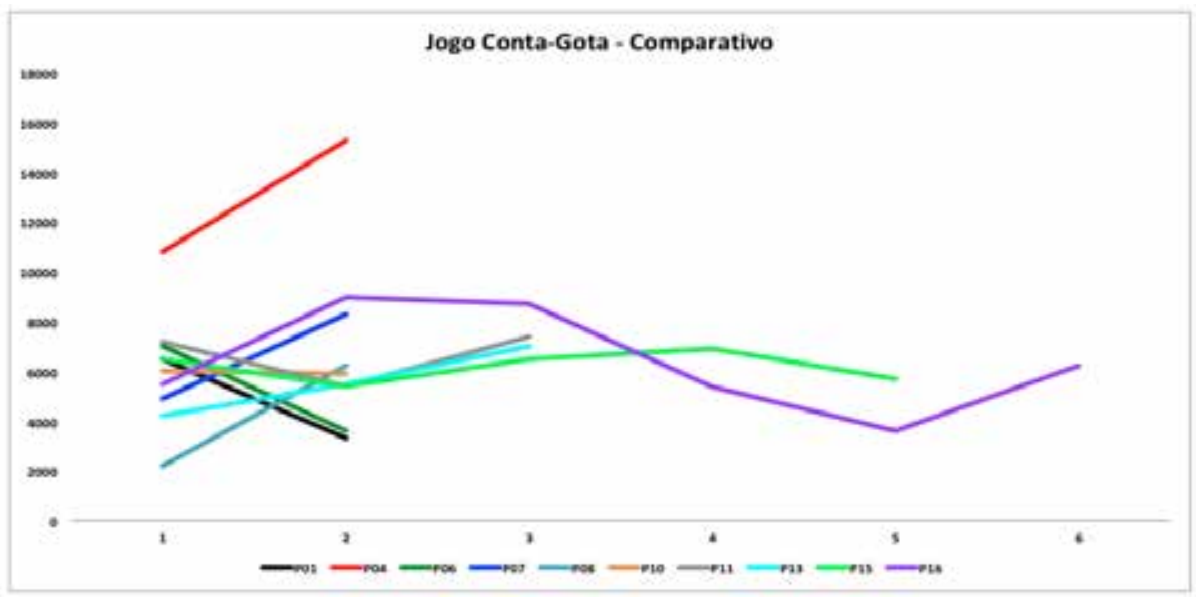

Ao analisar os resultados do Grupo A no Jogo Desafio do Quadro Negro (FIGURA 6), vê-se que o jogador P04 apresenta novamente os melhores resultados, ratificando que ele possui maiores habilidades para a matemática. De forma similar ao jogo anterior, o jogador P01 também demonstrou dificuldades nesse jogo. 
Figura 6

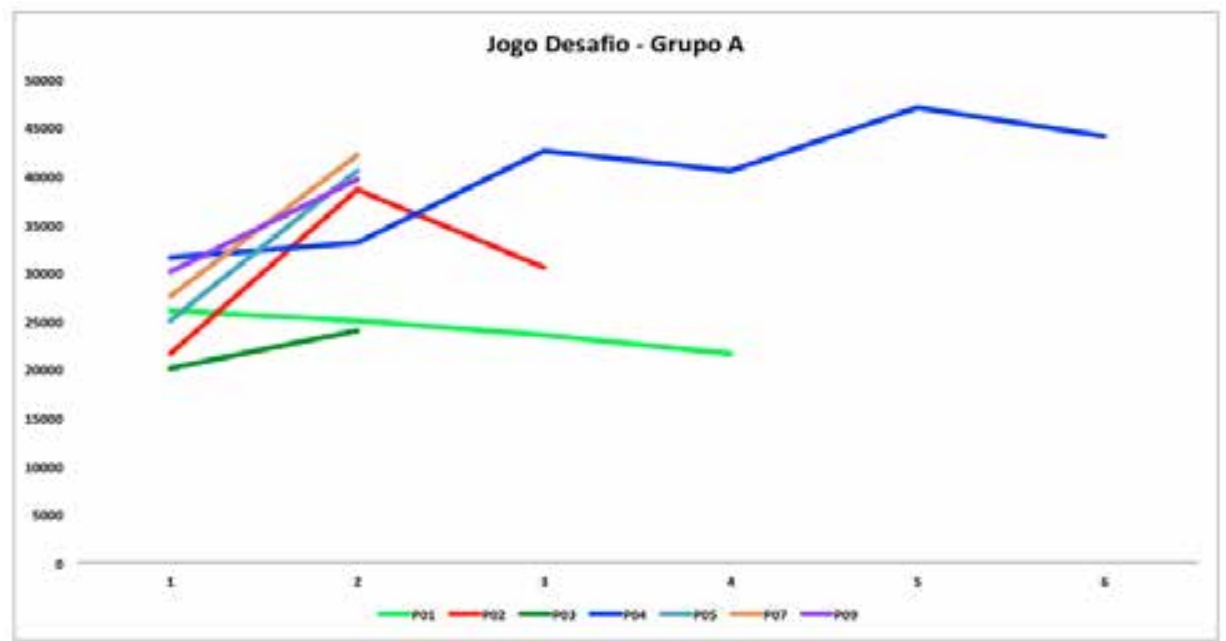

Já analisando o Grupo B (FIGURA 7), é possível observar, novamente, a instabilidade e uma certa regularidade entre os limites inferiores e superiores das pontuações dos jogadores, com exceção do jogador P14 que em suas interações apresentou valores sempre ascendentes.

\section{Figura 7}

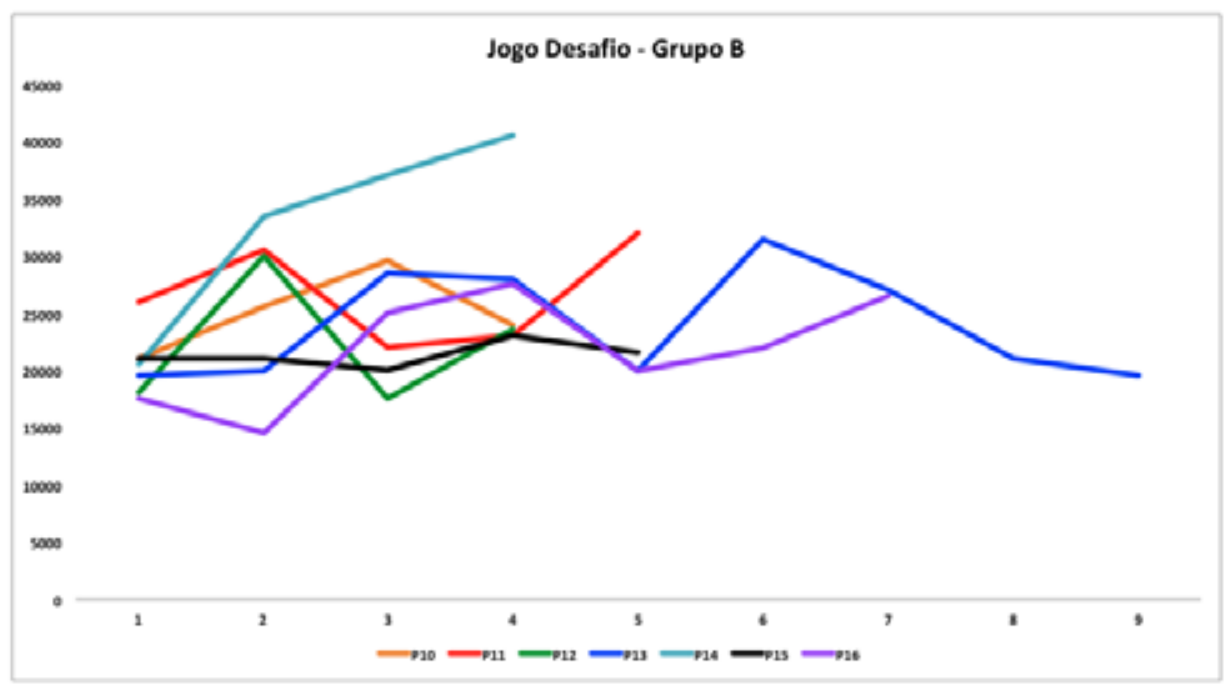


Vê-se que, comparando os dois grupos (FIGURA 8), os jogadores que mais se destacam são P04, P05, P07, P09, P14, apresentando os maiores escores durante suas interações. Os demais jogadores apresentaram características bem similares quanto as pontuações alcançadas. Analisando os jogadores com idades maiores P10, P12, P13 e P14, verifica-se que eles obtiveram na primeira interação pontuações mais baixas, algo que pode estar relacionado a falta de destreza com mecânica do jogo, mas essa diferença em suas idades não impactou no contexto geral os seus desempenhos, que foram similares aos jogadores mais jovens.

\section{Figura 8}

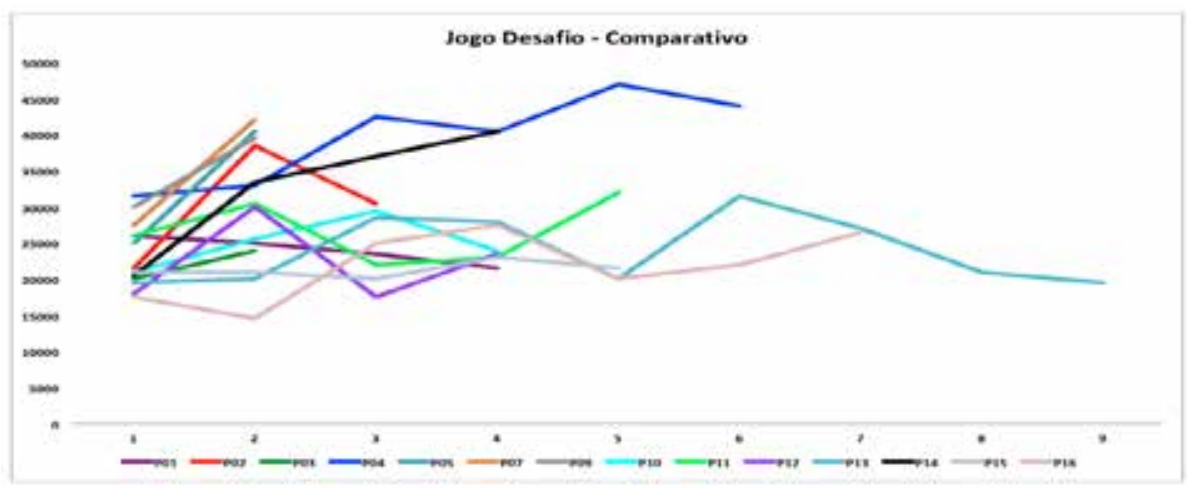

Diante dos dados obtidos nas interações dos jogadores e, relacionando o aumento da pontuação com a melhoria dos conhecimentos matemáticos, vê-se que grande parte dos alunos apresentaram uma constância em seus resultados, demonstrando que eles possuem um nível de conhecimento matemático basilar consolidado.

Esse conhecimento básico em aritmética é de profunda importância, pois como cita Sant'ana (2015), todos os outros ramos da matemática utilizam os princípios e as regras da aritmética e se estes princípios básicos não estiverem consolidados, ocorrerão problemas nas diversas aplicações da matemática.

Foi possível observar também que alguns alunos apresentaram pontuações crescentes, em especial o aluno P04, que demonstrou maiores conhecimentos em aritmética, enquanto outros alunos apresentaram pontuações mais baixas, sinalizando que eles podem possuir algum tipo de dificuldade em aritmética.

Como cita Sant'ana (2015), resolver problemas matemáticos, de simples a complexos, envolve manter oralmente ou por escrito em mente as diversas operações embutidas, relacionar os "sub-resultados” entre si e chegar ao resultado, por este motivo, calcular é uma das aprendizagens mais importantes e complexas do ser humano.

Nesse sentido, Fonseca (2017) sinaliza que para conseguir realizar todas essas etapas é necessário a todo momento recrutar diversos componentes das funções executivas para conseguir estabelecer pontes entre os conhecimentos e sistematizá-los e por este motivo precisamos estimular estas funções. 
Para esta mesma autora, quanto mais o indivíduo for exposto a um ambiente cognitivamente rico em contexto significativo social e emocionalmente, melhor será o cenário de desenvolvimento de sua memória de trabalho, de seu controle inibitório, de sua flexibilidade cognitiva, entre outras habilidades executivas. Desta forma, interagir diariamente com ambientes que estimulem estas habilidades podem fazer com que o aluno potencialize suas funções executivas.

Nesse contexto, estimular as funções executivas por meio dos jogos digitais pode ser uma boa intervenção, diante deste cenário tecnológico em que os jovens estão imersos e das contribuições que os jogos têm possibilitado. Como cita Sant'ana (2015), os jogos matemáticos podem auxiliar na formulação do pensamento aritmético, permitindo a produção de significados, possibilitando ao aluno lidar com suas frustações e ainda agir estrategicamente, trabalhando o lúdico e demais processos que intervém no ato de aprender.

Então, por meio da interação com estes digital brain games, que permitem o jogador acompanhar seus rendimentos e melhorias, como também de poder interagir mais vezes com jogos que estimulem as suas habilidades deficitárias, em seus treinos diários, podem conduzir o aluno a consolidar e aprimorar seus conhecimentos como também melhorar suas habilidades, como a memória e as outras funções executivas.

\section{CONCLUSÃO}

Ler, escrever e calcular são as aprendizagens mais importantes e complexas do ser humano e devem ser continuamente aprimoradas ao longo da vida. Diante desta complexidade, a aprendizagem matemática está associada aos maiores níveis de dificuldades na vida escolar e isso vem sendo apontado nas avaliações nacionais e internacionais.

A aritmética, como base para aprendizagem matemática, tem função essencial na aproximação do aluno à sua realidade cotidiana, já que ela é utilizada por nós a todo momento e se o aluno não possuir consolidada uma fundamentação aritmética, trará consigo conhecimentos fragmentados que dificultarão sua continuidade na aprendizagem escolar, como também em todo seu contexto social.

Como forma de reverter este quadro que evidencia a dificuldade dos alunos e intervir na base do conhecimento matemático dos estudantes universitários, estimular as funções executivas por meio da interação com os digital brain games pode permitir com que estes alunos consolidem e aprimorem seus conhecimentos de forma lúdica e desafiadora. Para tanto, os educadores são os principais modeladores e estimuladores das FE nos seus alunos, na medida em que fazem a mediação das múltiplas aprendizagens em suas aulas.

Assim, neste estudo, os resultados preliminares apontam que dos 16 alunos envolvidos na investigação, apenas 5 obtiveram os melhores resultados nos jogos, demonstrando que os demais apresentam um conhecimento aritmético intermediário ou apresentam dificuldades nesta área de conhecimento. Nosso intuito é que as próximas publicações possam trazer mais evidências das contribuições dos digital brain games para a aprendizagem matemática, como também de como a estimulação das funções executivas podem contribuir para estes fins. 


\section{REFERÊNCIAS}

ALTEMEIER, L., JONES, J., ABBOTT, R. D.; BERNINGER, V. W. Executive functions in becoming writing readers and reading writers: Note taking and report writing in third and fifth graders. Developmental neuropsychology, v. 29, n. 1, p. 161-173, 2006

ALVES, L. R. G. Práticas inventivas na interação com as tecnologias digitais e telemáticas: o caso do Gamebook Guardiões da Floresta. Revista de Educação Pública, v. 25, p. 574-593, 2016.

ALVES, Lynn.; BOMFIM, C. Gamebook e a estimulação de funções executivas em crianças com indicação de diagnóstico de TDAH: processo de pré-produção, produção e avaliação do software.

Revista FAEEBA: Educação e Contemporaneidade, v. 25, n. 46, p. 141-157, maio/ago. 2016.

BULL, R.; LEE, K. Executive functioning and mathematics achievement. Child Development Perspectives, v. 8, n. 1, p. 36-41, 2014.

CRAGG, L.; GILMORE, C. Skills underlying mathematics: The role of executive function in the development of mathematics proficiency. Trends in Neuroscience and Education, v. 3, n. 2, p. 6368, 2014.

DIAMOND, A. Executive functions. Annual review of psychology, v. 64, p. 135-168, 2013.

FONSECA, R. P. O papel das funções executivas (Especial Leitura e Escrita). NEUROEDUCAÇÃO, São Paulo, p. 42-50, 1 mar. 2017.

GONÇALVES, Hosana Alves et al. Funções executivas predizem o processamento de habilidades básicas de leitura, escrita e matemática? Neuropsicologia Latinoamericana, v. 9, n. 3, 2017.

MEGÍAS, P.; MACIZO, P. Simple arithmetic: electrophysiological evidence of coactivation and selection of arithmetic facts. Experimental Brain Research, v. 234, n. 11, p. 3305-3319, 2016.

OCDE. PISA 2015 Assessment and Analytical Framework: Science, Reading, Mathematic and Financial Literacy. Paris: OECD Publishing. 2016.

RAMOS, D. K.; ROCHA, N. L. Intervenção com jogos cognitivos em pequenos grupos no contexto escolar: contribuições às habilidades cognitivas. RETRATOS DA ESCOLA, v. 11, p. 277-294, 2017.

RIVERO, T. S. A construção de um método para o desenvolvimento de um videogame para treino de controle inibitório para adolescentes com Transtorno do Déficit de Atenção e Hiperatividade. 
2016. 124f. Tese (Doutorado) - Escola Paulista de Medicina, Universidade Federal de São Paulo, São Paulo, 2016.

RIVERO, T. S.; QUIRINO, E. H. G; STARLING-ALVES, I. Videogame: seu impacto na atenção, percepção e funções executivas. Neuropsicologia Latinoamericana, v. 4, p. 38-47, 2012.

ROCHA, P.; NERY, J.; ALVES, L. R. G. Jogos digitais e reabilitação neuropsicológica: delineando novas mídias. Seminário Tecnologias Aplicadas à Educação e Saúde, 1, Salvador. Anais..., UNEB, Campus I, 2014. p. 70-84.

RODRIGUES, C. C; RIVERO, T. S.; BERTALIA, Danila. O impacto do uso do videogame sobre o desenvolvimento cognitivo dos adolescentes. Revista Pandora, v. 30, p. 37-49, 2011.

SANT’ANA, N. A. S.; LAUDARES, J. B. Pensamento aritmético e sua importância para o ensino de matemática. Encontro Mineiro de Educação Matemática, 6, 2015. Anais..., São João Del Rei, 2015.

ST. CLAIR-THOMPSON, H. L.; GATHERCOLE, S. E. Executive functions and achievements in school: Shifting, updating, inhibition, and working memory. Quarterly Journal of Experimental Psychology, v. 59, 745-759, 2009.

TOURINHO, Amanda; BONFIM, C.; ALVES, L. Games, TDAH e Funções executivas: uma revisão de literatura Simpósio Brasileiro de Games e Entretenimento Digital, 15, 2016, São Paulo. Anais..., São Paulo: SBgames, 8 a 10 set. 2016.

VIEIRA, Jessica; CERQUEIRA, Larissa; DOREA, Maria de Fátima. Relatório de pesquisa Gamebook Guardiões da Floresta: interação no espaço escolar. Salvador: CNPq, 2016. 
1 Doutor e Mestre em Modelagem Computacional de Sistemas Cognitivos - SENAI/CIMATEC; Especialista em Educação, Comunicação e Tecnologias em Interfaces Digitais e em Metodologia do Ensino da Matemática; Graduado em Licenciatura em Matemática; Criador do site do Reforço Virtual de Matemática; Consultor Pedagógico na Área de Matemática na criação de jogos digitais; Professor da Graduação do Centro Universitário SENAI/CIMATEC. E-mail: william_tenor@yahoo.com.br

2 Doutora e mestra em Educação pela Universidade Federal da Bahia; Pós-doutora na área de Jogos eletrônicos e aprendizagem pela Università degli Studi di Torino, na Itália; Bolsista de Produtividade Desenvolvimento Tecnológico e Extensão Inovadora do CNPq - Nível 2. E-mail: Iynnalves@gmail.com

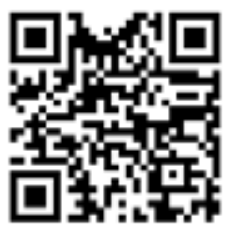

A autenticidade desse artigo pode ser conferida no site https://periodicos. set.edu.br

\section{(). (1) (2)}

Este artigo é licenciado na modalidade acesso abertosob a Atribuição-Compartilhalgual CC BY-SA

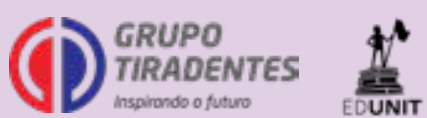

